\title{
Inmunodiagnóstico de la infección en humanos por Trypanosoma cruzi mediante ELISA utilizando sangre recolectada en papel de filtro
}

\author{
Luis C. Orozco', Diana Camargo², Myriam C. López ${ }^{3}$, Sofía Duque ${ }^{4}$, Luis E. Gualdrón", \\ Elvia Cáceres ${ }^{3}$, Margarita Ronderos ${ }^{5}$, Maritza Rey ${ }^{5}$, Augusto Corredor ${ }^{3}$.
}

\begin{abstract}
Resumen
Los estudios seroepidemiológicos para la detección de anticuerpos contra Trypanosoma cruzi requieren de un gran número de muestras y la obtención de sangre por punción venosa y su transporte se hacen difíciles y costosos. La recolección de sangre en papel de filtro minimiza éstas dificultades y el estudio valoró tanto éste sistema como la validez y reproducibilidad del inmunoensayo ELISA para el inmunodiagnóstico de la infección en humanos por $T$. cruzi. Se utilizó suero y eluídos de sangre recolectada en papel de filtro de personas de zona endémica de enfermedad de Chagas para la detección de anticuerpos contra $T$. cruzi mediante las pruebas de inmunofluorescencia indirecta (IFI) y ELISA. La validez del ELISA utilizando eluídos de sangre en papel de filtro presentó un área bajo la curva de receptor operador (ROC) de 0.9944. El acuerdo del ELISA entre los dos tipos de muestra presentó una distribución cercana a la normal con un promedio de -0.01 y una desviación estándar de 0.23 . Se evidenció que la reproducibilidad del IFI es inferior a la del ELISA. Esta mayor concordancia y la mayor sensibilidad y especificidad encontrada previamente para el ELISA hacen pensar en la posibilidad de presentarla como alternativa de prueba de referencia para la detección de anticuerpos contra $T$. cruzi y su utilización en estudios epidemiológicos.
\end{abstract}

Palabras clave: Chagas, eluído de sangre, ELISA, T. cruzi

\section{Immunodiagnosis of Trypanosoma cruzi infection in humans using ELISA on blood collected on filter paper}

\begin{abstract}
Seroepidemiological studies for the detection of Trypanosoma cruzi antibodies require a great number of samples; their collection through venous puncture and transport is difficult. Collection of blood on filter paper significantly reduces these problems to a minimum. This study assessed this system as well as the validity and reproducibility of the enzyme immunoassay (ELISA) for the diagnosis of Trypanosoma cruzi human infection. Serum samples and blood eluates collected on filter paper (taken from people living in zones where Chagas'disease is consideied to be endemic) were tested for Trypanosoma
\end{abstract}

1 Escuela de Enfermería, Universidad Industrial de Santander, Bucaramanga, Santander.

2 Escuela de Fisioterapia, Universidad Industrial de Santander, Bucaramanga, Santander.

3 Laboratorio de Parasitología, Instituto Nacional de Salud y Facultad de Medicina, Universidad Nacional de Colombia, Santafé de Bogotá, D.C., Colombia.

4 Laboratorio de Parasitología, Instituto Nacional de Salud, Santafé de Bogotá, D.C., Colombia.

5 División de Epidemiología, Ministerio de Salud Pública de la República de Colombia,Santafé de Bogotá, D.C., Colombia.

Recibido abril 20 de 1999 , aceptado junio 18 de 1999 
cruzi antibodies by using the immunofluorescent antibody test (IFAT) and ELISA. The validity of ELISA using blood eluates collected on filter paper showed an area under the receptor-operator curve (ROC) of 0.944. The agreement between both kinds of samples in the ELISA test showed a near to normal distribution with an average of -0.01 and a standard deviation of 0.23 . The reproducibility of the IFAT test was shown to be lower than that for the ELISA test. Its greater concordance, sensibility and specificity make the ELISA test a good alternative to the Trypanosoma cruzi antibody detection reference tests and for use in seroepidemiological surveys.

Key words: Chagas, blood eluates, ELISA, T. cruzi

\section{Introducción}

Los estudios seroepidemiológicos tienen una larga tradición que data desde mediados del siglo XX (1-3). Muchos de estos estudios fueron difíciles de realizar en el pasado debido a la complejidad de las técnicas serológicas que se utilizaban. El desarrollo de las nuevas tecnologías ha facilitado esta labor $(3,4)$, así como los sistemas simples de recolección de sangre (5).

Se ha recomendado que cuando se valore una técnica o un nuevo sistema de recolección de muestra de sangre, se realice tanto un estudio de validez como de reproducibilidad. El presente estudio seleccionó como referencia la prueba de inmunofluorescencia indirecta $(\mathrm{IFI})$ realizada en suero (6) para validar su reproducibilidad utilizando el inmunoensayo enzimático (ELISA) (7) comparando los resultados obtenidos con suero y eluidos de sangre seca recolectada en papel de filtro.

\section{Materiales y métodos}

Muestras. Se recolectaron al azar 595 muestras de sangre de personas de ambos géneros de diferentes edades, de zona rural y urbana de Guateque (Boyacá), de las que se obtuvieron sueros que fueron separados en alícuotas y almacenados $\mathrm{a}-20^{\circ} \mathrm{C}$.

Inmunofluorescencia indirecta. Se detectaron anticuerpos contra Trypanosoma cruzi en las muestras de suero (6) y eluídos de sangre seca en papel de filtro (5) mediante IFI. Esta sirvió como prueba de referencia, considerándose valores positivos los mayores o iguales a 1:32.

Ensayo inmunoenzimático ELISA. Se detectaron anticuerpos contra $T$. cruzi en las muestras de suero mediante ELISA siguiendo la metodología descrita por M. C. López y col. (8).

Recolección de muestras sobre papel de filtro. Se recolectaron al azar 595 muestras de sangre sobre papel de filtro Whatmann \#3, delimitando previamente un área circular con capacidad de absorción de $60 \mu \mathrm{l}$ de sangre. Las muestras se dejaron secar a temperatura ambiente sin exposición directa a la luz solar y, finalmente, se almacenaron a $4^{\circ} \mathrm{C}$.

Procesamiento. Para la obtención de suero, se recortó el círculo y se diluyć en $3 \mathrm{ml}$ de solución reguladora de fosfatos (PBS) durante 24 horas con lo cual se obtuvo una primera dilución equivalente a 1:100 de suero. A partir de éstas, se realizaron nuevas diluciones equivalentes a $1: 200,1: 400,1: 800,1: 1.600,1: 3.200$ con la finalidad de determinar la dilución óptima de la muestra para ser utilizada en la prueba de ELISA.

ELISA. La prueba inmunoenzimática se realizó usando el método descrito previamente por Corredor y col. (9).

Análisis. Para establecer la validez del ELISA utilizando sangre recolectada en papel de filtro, se realizó una curva del receptor operador (ROC) y se calculó el área bajo la curva (10), teniendo como prueba de referencia la IFI en suero y aceptando como positivo una dilución igual o mayor a 1:32.

Para evaluar la concordancia del ELISA entre la absorbancia del suero y el papel de filtro, se siguieron las recomendaciones de Bland y Altman (11), para variables continuas. Se evaluó la reproducibilidad para el IFI en suero y papel de filtro en la dilución 1:32, calculando la kappa de Cohen (12). 
Para el ELISA, se realizó la kappa (12) para comparar la reproducibilidad entre las pruebas realizadas en muestra de suero y eluidos de sangre de papel de filtro en los puntos de corte de absorbancias $0,3,0,4$ y 0,5 que son los puntos de mayor eficiencia (8).

\section{Resultados}

La validez del ELISA utilizando eluídos de sangre en papel de filtro para la detección de anticuerpos contra $T$. cruzi presentó un área bajo la curva ROC de 0,9944 como se observa en la figura 1.

La concordancia del ELISA utilizando el método de Bland y Altman (11) entre las absorbancias obtenidas entre los dos tipos de muestras, suero y eluídos de sangre recolectada en papel de filtro, para la detección de anticuerpos contra el parásito, se obtuvo una distribución cercana a la normal, con un promedio de $-0,01$ y una desviación estándar de 0,23 , lo cual produce unos límites de concordancia de $95 \%$ entre $-0,46$ y 0,44 . Los resultados generales se aprecian en la figura 2, donde las diferencias más amplias se encuentran en los sueros con absorbancias de valores superiores a 0,5 . Los sueros con valores de absorbancia entre 0,3 y 0,5 son relativamente pocos y los sueros con valores de absorbancia menores de 0,3 se agrupan, con muy poca dispersión, alrededor de 0 (cero) de diferencia.

Los resultados de concordancia para la comparación de $\mathrm{IFI} \geq 1: 32$ en suero y eluidos de sangre seca recolectados en papel de filtro y para

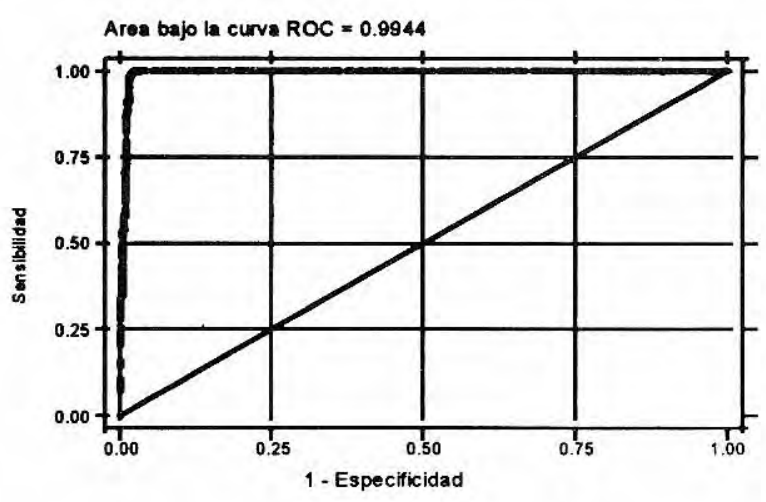

Figura 1. Area bajo la curva del receptor operador $(R O C)$ de la prueba de ELISA

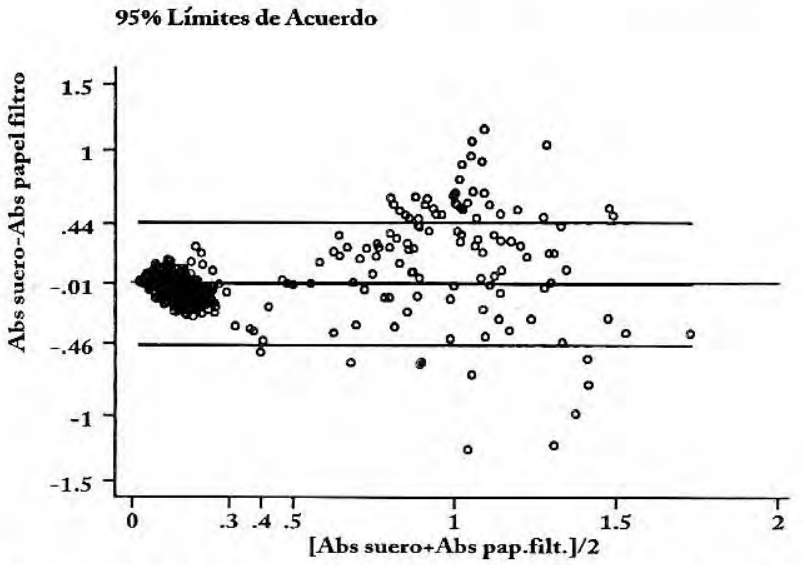

Figura 2. Límites de acuerdo del $95 \%$ utilizando el método de Bland y Altman entre las absorbancias del ELISA en suero y papel de filtro para la detección de anticuerpos contra Trypanosoma cruzi

el ELISA en los puntos de corte de absorbancia $0,3,0,4$ y 0,5 se muestran en el cuadro $1 a$ y $1 b$. Es notorio que la reproducibilidad de IFI es inferior a la del ELISA.

\section{Discusión}

La validez o exactitud, términos sinónimos, de una prueba se realiza cuando se compara una prueba nueva con una de referencia que, algunas

Cuadro 1a. Tabla tetracórica para el cálculo del kappa de Cohen

\begin{tabular}{cccc}
\hline & & \multicolumn{2}{c}{ Prueba A } \\
\hline & & Positiva & Negativa \\
Prueba & Positiva & a & b \\
B & Negativa & $c$ & d \\
\hline
\end{tabular}

Cuadro 1b. Valores de kappa para IFI y ELISA

\begin{tabular}{lcrrrr}
\hline Prueba A vs B & a & b & c & d & Kappa \\
\hline IFI dil. $\geq \mathbf{1 / 3 2}$ & 101 & 11 & 6 & 477 & 0,9049 \\
ELISA Abs. $\geq \mathbf{0 , 3}$ & 117 & 2 & 28 & 448 & 0,8544 \\
ELISA Abs. $\geq \mathbf{0 , 4}$ & 115 & 0 & 7 & 473 & 0,9631 \\
ELISA Abs. $\geq \mathbf{0 , 5}$ & 108 & 2 & 9 & 476 & 0,9401 \\
\hline
\end{tabular}

Prueba $A=$ suero; Prueba $B=$ papel de filtro

Concordancia para la comparación de $|F| \geq 1: 32$ en suero y eluidos de sangre seca recolectados en papel de filtro y ELISA en puntos de corte $0,3,0,4$ y 0,5 . 
veces, se la denomina prueba de oro, de referencia o diagnóstico. Para las pruebas dicotómicas, los valores más utilizados para establecer la validez o la exactitud son la sensibilidad y la especificidad; para pruebas con resultados continuos, es mejor utilizar toda la información calculando el área bajo la curva del receptor operador, ROC (8-10).

Al hablar sobre reproducibilidad y dependiendo de lo que se desee, se puede calcular la concordancia cuando se trata de variables nominales que clasifican a los individuos en grupos exhaustivos y excluyentes pero que no son categorías ordenadas, con la prueba kappa de Cohen (12). El caso más sencillo es la concordancia entre dos pruebas que clasifican los individuos en positivo y negativo.

Para el caso de variables continuas, a veces se desea conocer si una prueba o técnica recién estandarizada y evaluada en el laboratorio puede reemplazar a una ya existente. En este caso, los límites de concordancia de Bland y Altman (11) permiten establecer dichos límites, con los cuales la decisión no depende de los valores preestablecidos sino de la interpretación biológica que se deduzca de los resultados.

En los dos casos anteriores, concordancia y acuerdo, se mide la reproducibilidad.

Para que una técnica pueda ser utilizada rutinariamente en investigaciones seroepidemiológicas, debe demostrar no sólo su validez sino también su reproducibilidad. Además, debe ser sencilla, de bajo costo, rápida de realizar en el laboratorio y su interpretación no debe depender de interpretaciones subjetivas (3). Tanto la IFI como el ELISA cumplen estas condiciones, aunque se reconoce que la primera técnica mencionada tiene mayor componente de subjetividad.

Existen muy pocos trabajos en la detección de anticuerpos contra $T$. cruzi que comparen la reproducibilidad de la ténica estandarizada, aunque existen algunos que comparan entre técnicas.
El presente trabajo permite establecer que la reproducibilidad del IFI y el ELISA utilizando eluidos de sangre en papel de filtro son casi perfectas, según la clasificación que de los valores de kappa recomiendan Landis y Koch (13). Sin embargo, es importante mencionar que si se desea reemplazar el suero por eluidos de sangre seca recolectada en papel de filtro para la prueba de ELISA, solo sirve si se utiliza la técnica para definir positivo y negativo, porque los límites de acuerdo en especial con absorbancias mayores de 0,5 son muy amplios y, por tanto, si la prueba se desea utilizar para un seguimiento serológico no sería muy adecuada.

La mayor concordancia del ELISA encontrada en los puntos de corte 0,4 y 0,5 comparados con la que se obtienen para la IFI, así como la mayor sensibilidad y especificidad encontrada para esta técnica (8) hacen pensar en la posibilidad de discutir la posibilidad de reemplazo a la IFI por el ELISA como prueba de referencia para la detección de anticuerpos contra T. cruzi y para estudios epidemiológicos a gran escala.

\section{Agradecimientos}

El presente trabajo se realizó con la colaboración del personal científico y administrativo del Hospital San Rafael, Guateque, Boyacá.

\section{Referencias}

1. Evans AS. Epidemiology and the Public Health Laboratory. Am J Public Health 1967;57:1041-51.

2. Paul JR, White C. Serological epidemiology. New York: Academic Press; 1973.

3. Lobel HO, Kagan IG. Seroepidemiology of parasitic disease. Ann Rev Microbiol. 1978;32:329-47.

4. Maddison SE. Serodiagnosis of parasitic disease. Clin Microbiol Rev 1991;4:457-69.

5. de Souza SL, Camargo E. The use of filter paper blood smears in a practical fluorescent test for American trypanosomiasis serodiagnosis. Rev Inst Med Trop São Paulo 1966;8:254-60.

6. Camargo ME. Fluorescent antibody test for the serodiagnosis of American trypanosomiasis. Technical modification employing preserved culture forms of Trypanosoma cruzi in a slide test. Rev Inst Med Trop Sao Paulo 1966;8:227-34. 
7. Voller A, Bartlett A, Bidwell DE. Enzyme immunoassays for parasitic diseases. Tras R Soc Trop Med Hyg 1976;70:98106.

8. López MC, Duque S, Orozco LC, Camargo D, Gualdrón LE, Cáceres E, et al. Inmunodiagnóstico de la infección chagásica por ELISA. Biomédica 1999;19: 1-5.

9. Corredor A, López MC, Duque S, Nicholls RS. Estandarización y evaluación de ELISA en eluídos de sangre seca recolectada en papel de filtro para el diagnóstico de cisticercosis. Biomédica 1996;16:131-3.
10. Beck JR, Shultz EK. The use of ROC curves in test performance evaluation. Arch Pathol Lab Med 1986;110:13-20.

11. Bland JM, Altman DG. Statistical methods for assesing agreement between two methods of clinical measurement. Lancet 1986;y:307-10.

12. Fleiss JL. Statistical methods for rates and proportions. 2nd ed. New York: John Wiley \& Sons; 1981.

13. Landis J, Koch G. The measurement of observer agreement for categorical data. Biometrics 1977;33:159-74. 